\title{
Simple plantar ulcers treated by below-knee plaster and moulded double-rocker plaster shoe - a comparative study
}

\author{
D J PRING* \& N CASIEBANCA $\dagger$ \\ *Surgeon to Leprosy Mission Hospital, Anandaban, PO Box 151, \\ Kathmandu, Nepal; †Senior Medical Officer TLM Salur, India
}

Received for publication 16 December 1981

\begin{abstract}
Summary Fifty-five simple plantar ulcers in patients with Hansen's disease were treated in plaster for 6 weeks as outpatients. A conventional belowknee plaster of Paris (BK POP) was applied to 24 ulcers. Eighteen ulcers healed, 3 nearly healed and 3 failed to heal. Thirty-one ulcers were treated with a moulded double plaster of Paris (MD POP) shoe, 18 were healed, 8 almost healed and 5 failed to heal. The MD POP shoe was better accepted by patients and proved more economical; it also provides an acceptable and effective alternative to the BK POP for the outpatient treatment of simple plantar ulcers.
\end{abstract}

\section{Introduction}

Simple plantar ulcers heal rapidly with rest. The below-knee (BK) plaster has been used for many years as a means of providing rest for the ulcer to heal yet still allowing ambulation. ${ }^{1}$ It can be applied in the outpatient clinic and admission to hospital is not required. Using this method good results have been reported. ${ }^{2}$ However, plaster is both expensive and heavy; consequently a smaller plaster shoe has been designed which is cheaper and lighter. The results of this plaster shoe are compared with traditional BK plaster.

\section{Materials and methods}

Fifty-five simple plantar ulcers occurring in 47 patients suffering from Hansen's disease were admitted to the study. We defined a simple plantar ulcer as an ulcer on the plantar surface of the anaesthetic foot which did not involve either the underlying bone, joint or tendon. The patients were randomly divided into two groups. One group was treated with a conventional padded below-knee plaster, the second group with a padded moulded double-rocker plaster shoe. 
Plasters were applied over a small magnesium sulphate glycerine and acriflavine dressing to the ulcer and removed after 6 weeks. The majority of patients were treated as outpatients.

A moulded double-rocker plaster shoe consists of a double-rocker foot board which is applied under the foot. The arch is packed with wet plaster as a moulded platform around the foot to produce a shoe. It extends high enough up the foot to prevent removal but not above the malleoli so as to avoid pressure sores. Ankle movement is free. The toes are covered. Patients were instructed to walk with an elephant gait lifting their foot off the ground and replacing it flat rather than the usual walking pattern of heel strike, stance and push off, as this lessens the mechanical forces on the foot. Patients with foot drop, fixed inversion deformity, rocker bottom or short feet were excluded from the trial as these feet are not suitable for treatment with an MD POP shoe.

The site, size and depth of each ulcer were recorded as well as its duration, any previous ulcer at the same site and the state of the local skin. The ulcers were divided into deep ulcers involving muscle, and superficial ulcers involving only the subcutaneous fat. This division was rather subjective as often fibrous tissue had replaced the muscle and fat at the ulcer site.

After removal of plaster the ulcer site was inspected. If healed, patients were advised to resume full walking cautiously over the following week. Both at the time of application and removal of plasters intensive health education was given on foot care. Patients were taught the principles of daily foot inspection, foot soaking and scraping, and the importance of the regular wearing of soft-lined chappels.

\section{Results}

Thirty-one ulcers were treated with an MD POP shoe and 24 with BK POP. Of the 31 ulcers treated with MD shoe 18 (58\%) were fully healed after 6 weeks, $8(26 \%)$ were nearly healed (incomplete epithelial healing ulcer which rapidly healed within a few days of further rest) and $5(16 \%)$ failed to heal. Of the 24 treated by BK POP $18(75 \%)$ fully healed, $3(12.5 \%)$ were nearly healed and $3(12.5 \%)$ failed to heal.

The average duration of each ulcer prior to plaster application was 11.9 months (range 1 week to 7 years). Twenty-six (84\%) ulcers were either recurrent ulcers or had surrounding callous and scar, $21(87.5 \%)$ ulcers were first ulcers with good local skin. In 10 ulcers this information was not recorded. The distribution of the sites of each ulcer is shown in Fig. 1. The size and depth of ulcers is shown in Table 1. There was a greater preponderance of deep ulcers in the MD POP group.

Of the 11 ulcers which were nearly healed on removing plaster 9 were originally deep ulcers and only 2 superficial; 3 were size ' 1 ', 7 size ' 2 ' and 1 size ' 3 '. 

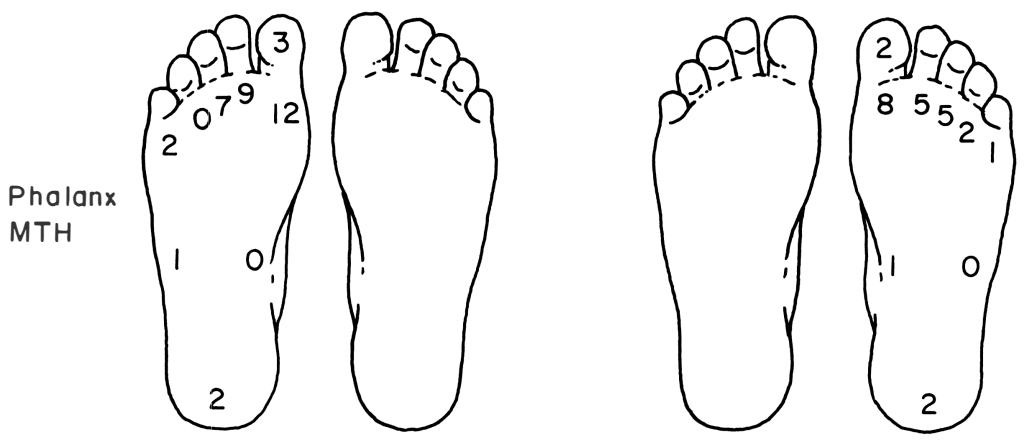

Figure 1. Distribution of ulcers. (NB some ulcers involved more than one site.)

Analysis of the 8 ulcers that failed to heal in 6 weeks reveals one superficial heel ulcer treated by MD POP shoe which was still superficial after 6 weeks. One superficial fore foot ulcer treated with MD shoe was healing after 6 weeks and the MD POP shoe was reapplied for a further 4 weeks after which the ulcer was healed. Another deep ulcer treated by MD shoe was healing but the patient refused further plaster and 5 months later the ulcer was still superficial.

Two ulcers, one treated by MD POP shoe and the other with BK POP had excessive granulation covering the ulcer and were treated with silver nitrate and healed.

One ulcer treated with BK POP was healing after 6 weeks but the patient was advised to have a multiple metatarsectomy to more evenly distribute the forces of weight bearing over the foot. Two ulcers, one treated with MD POP shoe the other with BK POP were probably misdiagnosed originally as simple ulcers when in fact they were complicated. Both were advised to have a dorsal incision and excision of the affected metatarsal heads.

\section{Discussion}

Simple plantar ulcers will heal rapidly if kept clean and rested. Because of the immense size of the problem it is neither practicable nor necessary to admit

Table 1. Size, depth and number of ulcers

\begin{tabular}{lcccccc}
\hline & \multicolumn{3}{c}{ Diameter* (no.) } & & \multicolumn{2}{c}{ Depth (no,) } \\
\cline { 2 - 3 } & 1 & 2 & 3 & & Superficial & Deep \\
\hline MD POP & 21 & 9 & 1 & & 11 & 20 \\
BK POP & 15 & 6 & 3 & & 13 & 11 \\
\hline
\end{tabular}

*Diameter: $1=0-1 \frac{1}{2}$ in.; $2=1 / 2-1$ in.; $3=1-2$ in. 
patients to treat these ulcers. Yet expecting a patient to stay at home resting in bed for several weeks is frequently impracticable as he is unable to earn a living for himself or his dependants.

Plaster treatment of simple plantar ulcers is a means whereby the patient can remain mobile while at the same time providing rest and a clean dressing for his ulcers. There are two criticisms of plaster treatment. First the patient may associate healing of his ulcer with plaster rather than with foot care and so he continues to reulcerate. We therefore at all times subject the patient to intensive health education regarding foot care. Secondly plaster immobilization results in disuse osteoporosis of bones, which renders them liable to fractures after plaster removal. Pain in the sensitive foot usually protects the osteoporotic foot from fracture and the normal patient limps for a few days after plaster removal. However, the patient with the anaesthetic foot has no such inhibitions so must be warned to gradually increase his daily walking and not immediately return to his old walking habits, risking both micro- and macro-fractures.

The BK POP immobilizes the foot more than the MD POP shoe which does not immobilize the ankle, subtalar or mid-tarsal joints which probably explains the difference in results with ulcers treated by BK POP rather than the MD shoe. However, the results using the MD shoe are not that much inferior $87.5 \%$ compared with $84 \%$ when the healed and nearly healed are grouped together. The MD shoe is more acceptable to the patient, cheaper to apply and, more important, likely to cause less disuse osteoporosis than the BK POP and therefore the risks of fracture should be reduced.

Too few heel ulcers were included in the study to conclude how effective the MD POP boot is in healing these difficult ulcers.

\section{References}

1 Fritischi EP. Care of the foot. In: Thangaraj RH, ed. A manual of leprosy 2nd edn Delhi: The Leprosy Mission, 1980; ch. 15.

2 Price EW. Lepr Rev, 31.3 .1960$. 